\title{
Fatores associados à não adesão ao tratamento para tuberculose pulmonar
}

Greyce Khoury Mansour ${ }^{1}$ (D), Luísa de Paula Quintanilha Ferreira ${ }^{1}$ (D), Gabriella de Oliveira Martins ${ }^{1}$ (D), Jessica Luanda Lemos Melo ${ }^{1}$ (D), Patrícia Scotini Freitas ${ }^{1}$ (D), Murilo César do Nascimento ${ }^{1}$ (D)

\begin{abstract}
RESUMO
Objetivo: analisar as evidências disponíveis na literatura sobre os fatores relacionados à não adesão ao tratamento diretamente observado por pessoas com tuberculose pulmonar. Métodos: trata-se de uma revisão integrativa da literatura. A busca ocorreu entre os meses de agosto de 2019 e maio de 2020, por acesso às bases de dados MEDLINE, LILACS, BDENF, BINACIS, IBECS, PUBMED, SCOPUS, e WEB OF SCIENCE, bem como via biblioteca virtual SCIELO, selecionando artigos publicados entre 2009 e 2019. Resultado: dos 1664 estudos pré-analisados foram identificados e selecionados 57 artigos que atenderam à pergunta de pesquisa. Após a extração dos dados, os fatores de não adesão foram estratificados em cinco categorias: fatores relacionados ao paciente, aspectos socioeconômicos, fatores referentes ao tratamento, fatores relacionados aos profissionais/serviço de saúde e aspectos de conhecimento, os quais apresentaram uma correspondência de 49, 33, 41, 23 e 20 artigos, respectivamente. Conclusão: Este estudo possibilitou analisar as evidências científicas relacionadas aos fatores da não adesão ao tratamento diretamente observado por pessoas com tuberculose pulmonar. Conclui-se que a multifacetada gama de aspectos associados a tal problema de pesquisa pode ser sintetizada em fatores relacionados ao paciente, a aspectos socioeconômicos, a fatores referentes ao tratamento, fatores relacionados aos profissionais/serviço de saúde e a aspectos inerentes ao conhecimento.
\end{abstract}

Palavras-chave: Cooperação e adesão ao tratamento, Terapia diretamente observada, Tuberculose pulmonar, Revisões sistemáticas como assunto.

\section{INTRODUÇÃO}

Tuberculose (TB) é uma doença infecciosa causada pelo bacilo Mycobacterium tuberculosis, cuja transmissão ocorre pelo ar, quando a pessoa infectada pelo bacilo o expele durante a fala, tosse ou espirro ${ }^{1,2}$. De acordo com o relatório de 2018 da World Health Organization (WHO) ${ }^{3}$, a TB está entre as dez principais causas de morte no mundo, sendo responsável, em 2017, por aproximadamente 1,3 milhão de mortes. A WHO avalia também que 30 países, incluindo o Brasil, concentram $87 \%$ de todos os casos no mundo ${ }^{3}$.

O tratamento para a TB atualmente preconizado é à base de antibióticos, com duração estimada de seis meses que inclui normalmente 0 uso de quatro medicamentos de primeira linha: rifampicina, isoniazida, pirazinamida e etambutol $^{3}$. A estratégia DOTS (Tratamento Diretamente
Observado de Curta Duração) é recomendada em todo o mundo por garantir melhores resultados de tratamento. Caso a tomada dos medicamentos seja observada pelo menos três vezes por semana na primeira fase e duas vezes por semana durante a fase de manutenção, pode-se considerar o tratamento em questão como diretamente observado. O tratamento preconizado, embora muito eficaz, não recebe adesão de todos os pacientes ${ }^{4,5,6}$. De acordo com Brasil (2011) ${ }^{4}$, quando o paciente deixa de comparecer, após a data prevista para seu retorno, por pelo menos 30 dias seguidos, considera-se abandono do tratamento.

O abandono do tratamento é um problema de grande importância para o controle da TB, pois tem como consequências a persistência do agente infeccioso, o aumento das taxas de recidiva, assim como da mortalidade, além de proporcionar maior chance de desenvolvimento de cepas

${ }^{1}$ Universidade Federal de Alfenas, UNIFAL- (MG) 
de bacilos resistentes ao tratamento e contribuir para a manutenção da cadeia de transmissão ${ }^{7}$. Apesar de a WHO preconizar que, para o controle da doença, a meta de cura seja igual ou superior a $85 \%$ e que a de abandono seja menor do que $5 \%$, as taxas de adesão não ultrapassam $40 \%$ em países em desenvolvimento ${ }^{3,8}$. Em 2014, o índice de abandono do tratamento no Brasil foi de $11,3 \%{ }^{9}$. Ao se considerar diferentes países, têm-se porcentagens de não adesão variadas, sendo de $35 \%$ na África do Sul $^{10}$ e $25,3 \%$ na China ${ }^{11}$.

Diante do exposto, o objetivo deste estudo foi analisar as evidências disponíveis na literatura sobre os fatores relacionados a não adesão ao tratamento diretamente observado pelas pessoas com tuberculose pulmonar.

\section{MÉTODO}

\section{Tipo de Estudo}

Este estudo tratou-se de uma revisão integrativa da literatura, realizada com o propósito de adensar o conhecimento científico/profissional referente ao tema de interesse. A revisão integrativa constitui-se em um método científico cuja finalidade é a de sintetizar resultados obtidos em estudos sobre determinado tema/questão, "de maneira sistemática, ordenada e abrangente"12.

O estudo contemplou as seis etapas de uma revisão integrativa que, segundo Mendes, Silveira e Galvão (2008) ${ }^{13}$, são: 1 ) identificação do tema e definição da questão de pesquisa; 2 ) estabelecimento de critérios para inclusão e exclusão de estudos/busca na literatura; 3) definição das informações a serem extraídas dos estudos selecionados/categorização dos estudos; 4) avaliação dos estudos incluídos; 5) interpretação dos resultados; 6) apresentação da revisão/síntese do conhecimento.

Para a elaboração da questão de pesquisa, recorreu-se à estratégia PICO, que representa um acrônimo para Paciente, Intervenção, Comparação e Outcomes (desfecho). Apesar de essa estratégia ser mais empregada nas revisões sistemáticas com o enfoque das pesquisas clínicas, a escolha da PICO para esta revisão integrati- va se deu em vista da compreensão de que os quatro componentes nela abordados constituem elementos fundamentais da questão de pesquisa no contexto da Prática Baseada em Evidências $(\mathrm{PBE})^{14}$. Assim, obteve-se a seguinte pergunta norteadora: Quais são as evidências disponíveis na literatura sobre os fatores relacionados com a não adesão ao tratamento diretamente observado pelas pessoas com tuberculose pulmonar?

\section{Critérios de inclusão}

Quanto ao critério de inclusão, ficou pré-fixado que os estudos a serem incluídos deveriam constituir artigos originais (estudos primários), cujo objeto de estudo fosse a abordagem a não adesão referente ao tratamento diretamente assistido para a tuberculose pulmonar, publicados entre 2009 e 2019, nos idiomas português, inglês e espanhol, em portais/bibliotecas e em bases de dados nacionais e internacionais. Já como critério de exclusão, considerou-se como não elegíveis os estudos que não respondam à questão de pesquisa, artigos de revisão e estudos que abordassem tratamentos diferentes ao TDO.

A busca na literatura aconteceu entre os meses de agosto de 2019 e maio de 2020, através de acesso às bases de dados: MEDLINE, LILACS, BDENF (Base de Dados em Enfermagem), BINACIS (Bibliografia Nacional em Ciências da Saúde Argentina), IBECS (Índice Bibliográfico Espanhol de Ciências de Saúde), que foram consultadas via Portal BVS (Biblioteca Virtual da Saúde), PUBMED, SCOPUS, e WEB OF SCIENCE, bem como à biblioteca virtual SCIELO (Scientific Eletronic Library Online).

\section{Descritores}

Os descritores controlados utilizados na pesquisa foram: $A=$ Treatment Adherence and Compliance/ Cumplimiento y Adherencia al Tratamiento/ Cooperação e Adesão ao Tratamento; $B=$ Directly Observed Therapy/ Terapia por Observación Directa/ Terapia Diretamente Observada; e $\mathrm{C}=$ Tuberculosis Pulmonary/ Tuberculosis Pulmonar/ Tuberculose Pulmonar. Utilizaram-se os des- 
critores nos idiomas português, inglês e espanḩol para as bases consultadas via Portal BVS, conforme o dicionário DECS, bem como os descritores em inglês para as consultas às bases PUBMED, SCOPUS, e WEB OF SCIENCE, segundo o dicionário MESH.
Nos cruzamentos entre os descritores controlados nos três idiomas de interesse, utilizou-se o operador booleano "AND", seguindo as combinações A x B, A x C e B x C. O Quadro 1 apresenta o número de artigos identificados em cada base de dados como primeiro resultado das buscas.

Quadro 1. Número de artigos primariamente identificados na estratégia de busca.

\begin{tabular}{|c|c|c|c|}
\hline \multirow{2}{*}{$\begin{array}{l}\text { Cruzamentos } \\
\text { Descritores } \\
\text { (DECS e MESH) }\end{array}$} & \multicolumn{2}{|c|}{$\begin{array}{l}\text { No de artigos identificados nas bases de dados } \\
\text { (por idioma do descritor) }\end{array}$} & \multirow[b]{2}{*}{ Espanhol } \\
\hline & Português & Inglês & \\
\hline $\begin{array}{l}\text { A x B } \\
\text { Cooperação e Adesão } \\
\text { ao Tratamento } \\
\text { X } \\
\text { Terapia Diretamente } \\
\text { Observada }\end{array}$ & $\begin{array}{l}\text { BDENF (1) } \\
\text { BINACIS (2) } \\
\text { IBECS(0) } \\
\text { LILACS (3) } \\
\text { MEDLINE (33) } \\
\text { SCIELO (3) } \\
\text { SCOPUS (1) }\end{array}$ & $\begin{array}{l}\text { BDENF (5) } \\
\text { BINACIS (2) } \\
\text { IBECS (5) } \\
\text { LILACS }(12) \\
\text { MEDLINE (320) } \\
\text { PUBMED (474) } \\
\text { SCIELO (3) } \\
\text { SCOPUS (16) } \\
\text { WEB OF SCIENCE (31) }\end{array}$ & $\begin{array}{l}\operatorname{BDENF}(0) \\
\text { BINACIS(0) } \\
\text { IBECS (1) } \\
\text { LILACS(0) } \\
\text { MEDLINE (2) } \\
\text { SCIELO (0) }\end{array}$ \\
\hline $\begin{array}{l}\text { A x C } \\
\text { Cooperação e Adesão } \\
\text { ao Tratamento } \\
\text { X } \\
\text { Tuberculose Pulmonar }\end{array}$ & $\begin{array}{l}\text { BDENF(1) } \\
\text { BINACIS (2) } \\
\text { IBECS(0) } \\
\text { LILACS (7) } \\
\text { MEDLINE (56) } \\
\text { SCIELO (1) }\end{array}$ & $\begin{array}{l}\text { BDENF (5) } \\
\text { BINACIS (2) } \\
\text { IBECS (3) } \\
\text { LILACS (19) } \\
\text { MEDLINE (257) } \\
\text { PUBMED (510) } \\
\text { SCIELO (4) } \\
\text { SCOPUS (4) } \\
\text { WEB OF SCIENCE (37) }\end{array}$ & $\begin{array}{l}\operatorname{BDENF}(1) \\
\operatorname{BINACIS}(0) \\
\operatorname{IBECS}(2) \\
\text { LILACS (4) } \\
\text { MEDLINE (4) } \\
\text { SCIELO (2) }\end{array}$ \\
\hline $\begin{array}{l}\text { B x C } \\
\text { Terapia Diretamente } \\
\text { Observada } \\
\text { X } \\
\text { Tuberculose Pulmonar }\end{array}$ & $\begin{array}{l}\text { BDENF (2) } \\
\text { BINACIS (3) } \\
\text { IBECS(0) } \\
\text { LILACS (9) } \\
\text { MEDLINE (172) } \\
\text { SCIELO (1) } \\
\text { SCOPUS (1) }\end{array}$ & $\begin{array}{l}\text { BDENF (4) } \\
\text { BINACIS (3) } \\
\text { IBECS (3) } \\
\text { LILACS (18) } \\
\text { MEDLINE (391) } \\
\text { PUBMED (449) } \\
\text { SCOPUS (456) } \\
\text { SCIELO (10) } \\
\text { WEB OF SCIENCE (211) }\end{array}$ & $\begin{array}{l}\text { BDENF (2) } \\
\text { BINACIS (3) } \\
\text { IBECS }(0) \\
\text { LILACS (9) } \\
\text { MEDLINE (172) } \\
\text { SCIELO (1) }\end{array}$ \\
\hline
\end{tabular}

Fonte: os autores (2020)

Os resultados das buscas foram salvos e exportados gradualmente nos diferentes formatos/extensões de arquivos digitais disponíveis em cada busca, para subsequente importação para o Rayyan, um aplicativo/website online gratuito, útil para auxiliar pesquisadores no desenvolvimento de revisões sistemáticas ${ }^{15}$.

O conjunto de arquivos importados para - Rayyan totalizou 3755 artigos, incluindo as referências repetidas de todas as bases de dados consultadas e os cruzamentos possíveis realizados. Destes, 872 registros foram excluídos de forma automática pelo aplicativo por serem artigos repetidos da mesma base de dados, resultando em 2881. Além disso, dois artigos não foram encontrados pelo Rayyan por possuírem um formato diferente dos aceitos pelo programa. Desses, 1997 foram consideradas pelo Rayyan 
duplicidades e 884 registros como não duplicidades. A atenção dos pesquisadores foi necessária para resolver de forma pontual cada uma das situações de aparente ou de real duplicidade. Desse modo, após exclusões manuais e automáticas, 1217 artigos foram descartados, resultando num conjunto de 1664 artigos pré-selecionados para a revisão (Figura 1 ).

\section{Análise das evidências}

A partir de então, o orientador da pesquisa designou a função de avaliação às cegas para três autoras, através do recurso "Blind on", disponível no Rayyan, momento disparador para a avaliação do título e resumo dos 1664 artigos pré-selecionados. Realizadas as avaliações quanto à inclusão ou não dos artigos na revisão, foi possível identificar divergência em $8,71 \%$ das decisões. Tais conflitos foram resolvidos com a descontinuidade do cegamento inicial, com a reavaliação conjunta dos 145 artigos com avaliação inicial heterogênea e com o consenso final sobre a inclusão/exclusão dos trabalhos revisitados. Ao final do processo, as revisoras consideraram que 1583 trabalhos não continham elementos que respondessem à questão norteadora do presente estudo e que outros 24 trabalhos não respondiam aos critérios de ano de publicação e de idiomas pré-selecionados, sendo incluído na revisão um conjunto de 57 artigos.

Para a definição das informações a serem extraídas dos estudos, utilizou-se uma versão adaptada do instrumento de Ursi $(2005)^{16}$ que contou com características referentes a: identificação, instituição sede/cenário do estudo, tipo de revista científica, características metodológicas do estudo, avaliação do rigor metodológico. Já para a análise e posterior síntese dos artigos incluídos, utilizou-se um quadro resumo, também inspirado no de Ursi $(2005)^{16}$, que conteve os seguintes campos para preenchimento: título do artigo, autores, nome do periódico, ano de publicação, instituição sede/cenário do estudo, resultados (fatores relacionados à não adesão ao TDO e fatores relacionados ao abandono do TDO) e conclusão.

$\mathrm{Na}$ sequência, mantendo o alinhamento com as etapas descritas por Mendes, Silveira e Galvão (2008) ${ }^{13}$, procedeu-se à avaliação dos estudos incluídos. Como parte da análise crítica dos artigos em relação à qualidade das evidências, adotou-se a classificação de Stillwell et al. $(2010)^{17}$ que aborda sete níveis de evidência.

Após a avaliação dos estudos, prosseguiu-se a interpretação dos resultados e a apresentação da revisão/síntese do conhecimento. Para tanto, realizou-se leitura exaustiva dos artigos, sendo adotada a técnica de análise temática de Minayo $(2010)^{18}$ para categorizar os fatores de abandono e de não adesão ao TDO, conforme apresentado a seguir.

\section{RESULTADOS}

Considerando os 57 artigos selecionados, 2017 foi o ano que apresentou maior número de publicações dessa temática em relação aos outros pesquisados, com um total de dez artigos publicados. Os resultados das buscas, bem como o processo de seleção, foram sintetizados no fluxograma a seguir. 
Figura 1. Fluxograma das buscas dos estudos.

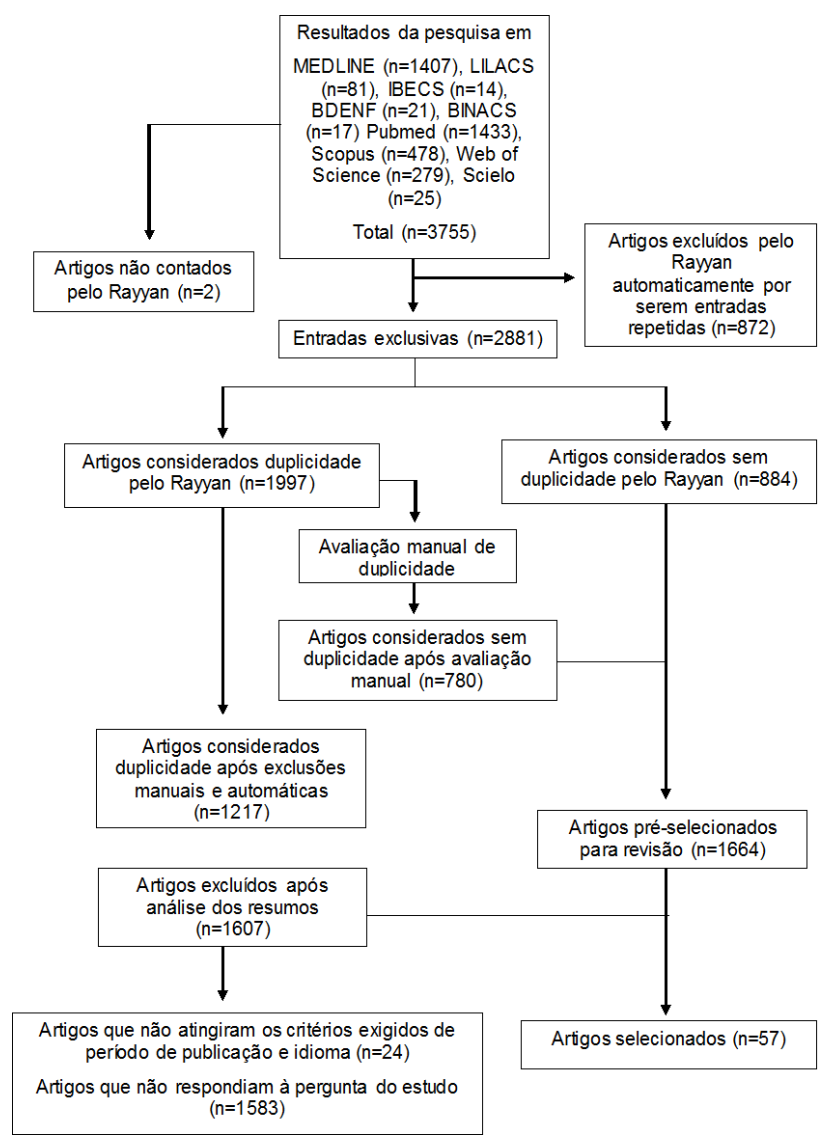

Fonte: os autores (2020)

De acordo com o nível de evidência, um artigo foi incluído na categoria II (estudo randomizado controlado), 45 foram classificados como IV (estudo caso-controle ou estudo de coorte) e 11 como VI (estudo qualitativo ou descritivo). Nesse contexto, é perceptível que a maioria dos artigos se encontrou no nível IV de evidência, ou seja, artigos com metodologia observacional analítica e não experimental. Parcela expressiva desses artigos remetia a estudos quantitativos em que foi privilegiada a coleta de dados por inquéritos a pessoas diagnosticadas com tuberculose e aos profissionais que lhes prestavam assistência à saúde.

Para melhor visualização e compreensão dos fatores de não adesão encontrados na literatura, optou-se por agrupá-los em cinco categorias, conforme a análise dos seus conteúdos. Tomados em conjunto, os fatores de não adesão foram: fatores relacionados ao paciente, aspectos socioeconômicos, fatores referentes ao tratamento, fatores relacionados aos profissionais/serviço e aspectos de conhecimento.

Os fatores de não adesão relacionados ao paciente configuraram: etilismo, tabagismo, coinfecção por HIV e crenças (não se sentir doente), presentes em 49 artigos - 85,96\% dos trabalhos. Quanto aos aspectos socioeconômicos abordados em 33 trabalhos (57,89\%), identificaram-se os fatores: baixa escolaridade, custos do tratamento (medicação e deslocamento), baixo nível socioeconômico, desemprego ou emprego informal, desnutrição, analfabetismo e moradias com infraestrutura precária. Sobre os fatores referentes ao tratamento abordados em 41 artigos $(71,92 \%)$, foram registrados: estigma, logística, retratamento e duração/efeitos adversos da DOT. Como fatores relacionados aos profissionais e ao serviço, despontaram-se: dificuldades na interação entre o profissional e o paciente, falta de estrutura e falta de preparo (segundo 23 artigos - 40,35\% dos trabalhos). Por fim, os aspectos de conhecimento (presentes em 35\% dos artigos - 20 trabalhos) foram a falta de conhecimento sobre a doença e a falta de conhecimento sobre o tratamento. Tal conjunto de achados é discutido a seguir.

\section{DISCUSSÃO}

\section{Fatores relacionados ao paciente}

Essa categoria foi a mais encontrada nos artigos analisados ${ }^{19-67}$. O etilismo, o tabagismo e a coinfecção por HIV foram os fatores de abandono mais mencionados. Outros fatores incluem ter idade avançada, ser do sexo masculino e ter crenças dificultadoras (o paciente sentir que não está mais doente).

Observam-se divergências no que diz respeito a sexo, etnia e faixa etária. Embora todos os artigos tenham analisado esses fatores, apenas seis deles consideraram a questão do sexo como um fator de abandono, que em todos foi maior no sexo masculino $27,29,33,43,59,63$. Apenas dois artigos consideraram a etnia preta e amarela como fatores de não adesão ${ }^{57,59}$. Somente sete estudos concluíram que a faixa etária estaria relacionada 
ao abandono do tratamento, entretanto não houve consenso em relação às idades, já que dois deles relacionaram a não adesão à faixa etária dos 20 aos 39 anos $^{42,59}$; outros dois artigos afirmaram que ser jovem era um fator de não adesão ${ }^{38,39}$; em um dos artigos ela estava relacionada à idade avançada ${ }^{31}$; em outro, ela era maior em pacientes menores de 35 anos $^{65}$ e, por fim, um artigo apontou a faixa etária de 20 a 29 anos como a relacionada ao abandono do tratamento ${ }^{57}$.

Um estudo do Jornal Mundial de Pesquisa Farmacêutica, feito na Índia, não achou resultados estatisticamente significantes quanto a variáveis como gênero, idade, estado civil e residência ${ }^{68}$, estando de acordo com os outros 37 artigos enquadrados nessa categoria ${ }^{19-26,28,30,32,34-37,40,41,44-56,58,60-62,64,66,67}$.

Sobre a questão do etilismo, tabagismo e coinfecção por HIV, a maioria $^{20,22,24-31,37-40,42,43,46-48,51,53,55,57-62,64,65}$ dos artigos que analisou estas variáveis concluiu que se trata de fatores relacionados ao abandono do tratamento. Esses achados condizem com o visto em outras literaturas mais recentes ${ }^{69-71}$.

\section{Aspectos Socioeconômicos}

Os aspectos socioeconômicos mais encontrados dizem respeito à baixa escolaridade e gastos com tratamento, incluindo custos com medicamentos e/ou custos com deslocamento até o centro de tratamento, e ao baixo nível socioeconômico. Outros aspectos mencionados dizem respeito a estar desempregado ou a ter emprego informal, desnutrição e analfabetismo.

$\mathrm{Em}$ todos os artigos em que a questão socioeconômica foi estuda-

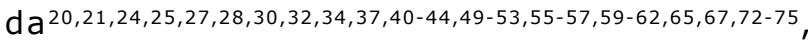
apontou-se o baixo nível socioeconômico como causa. Outros fatores muito importantes que levam ao abandono, também muito menciona$\operatorname{dos}^{20,21,24,27,28,32,37,40-44,50-52,55,57,59-62,65,67,72,73,75}$, são a baixa escolaridade, o desemprego, a baixa renda mensal e os gastos com o tratamento. Esses gastos envolvem desde a compra dos medicamentos, dependendo do país em que se está tratando, até os custos com deslocamento para os centros de saúde. Como são meses de tratamento, a baixa renda e o desemprego tornam-se questões importantes que merecem destaque dentre os fatores de não adesão. Esses fatores estão de acordo com o encontrado na literatura68,71.

Dentre os estudos mais atuais, há um realizado no Brasil que aborda a questão social no tratamento da TB com uma análise qualitativa a partir da experiência de profissionais de saúde. Essa pesquisa mostra a associação entre TB e vulnerabilidade social, como uso de álcool e de drogas ilícitas, baixa renda e dificuldade de deslocamento até unidades de saúde, fatores que condizem com os encontrados nos resultados apresentados. Além disso, a pesquisa reforça a influência positiva de programas como o Bolsa-Família e fornecimento de vale-transporte para auxiliar na adesão ao tratamento ${ }^{76}$.

Em um estudo conduzido no Brasil e publicado em 201977, dois grupos de pessoas, ambos com TB foram analisados: um deles recebia auxílio financeiro do governo e o outro, não. Esse estudo concluiu que aquele grupo assistido pelo governo obteve uma taxa de cura $8 \%$ maior do que o outro.

\section{Fatores Referentes ao Tratamento}

Nessa categoria, se sobressaíram os seguintes fatores: estigma, logística, retratamento, duração do tratamento e seus efeitos adversos. Dentre os efeitos adversos, os mais prevalentes são a artralgia e os gastrointestinais, como náusea, vômito e epigastralgia, o que leva muitos pacientes a abandonarem o tratamento da doença. A duração também é um fator de grande influência na não adesão, visto que são, usualmente, seis meses de tratamento. Em relação aos pacientes previamente tratados para tuberculose, constatou-se que grande parte deles não realizava o retratamento.

Um fator importante e muito ressaltado pelos artigos que se enquadraram nessa categoria ${ }^{19-21,23-25,28-30,33-38,40,41,43-46,48,49,51,52,54-56,60-66,72-74,78-80}$ é a logística. Muitas pessoas abandonam o tratamento devido à longa distância entre a residência e o centro de saúde. Como os serviços de saúde costumam ser centralizados, os pacientes que moram em regiões mais afastadas, a dez ou mais 
quilômetros de distância dos centros, tendem a abandonar o tratamento. Essa questão é bastante elucidada na literatura e confirma o encontrado neste estudo ${ }^{6,71}$.

Em um artigo brasileiro, observou-se que a descentralização dos centros para a atenção primária, envolvendo a Estratégia Saúde da Família (ESF) na cidade de Curitiba, no Paraná, resultou em aumento do número de pessoas atendidas e em redução da proporção de abandono. Esse artigo também destaca a importância da ESF como porta de entrada para o sistema de saúde e seu papel fundamental no acolhimento do paciente ${ }^{81}$.

A questão do estigma merece destaque, pois, em todos os artigos em que essa variável foi analisada $20,21,30,32,35,36,43,54-56,66$, foi enquadrada como um fator de não adesão. O estigma existente em relação à doença é motivo de vergonha do paciente que muitas vezes tenta esconder a doença da comunidade, e, por isso, acaba não se tratando. Muitos pacientes reportam medo de perderem amigos e familiares em decorrência do estigma existente em relação à doença e às condições que a permeiam. Todas essas questões desencadeadas pelo estigma acabam impactando consideravelmente a qualidade de vida desses pacientes. Por isso, é importante explicar para os familiares e para o próprio paciente sobre a doença e seu tratamento, para que ele possa receber apoio familiar e não abandone o tratamento.

Um estudo de Linhares e Paz (2020) ${ }^{82,}$ conduzido em unidades Estratégia Saúde da Família (ESF), no município do Rio de Janeiro, concluiu que, devido ao estigma, o paciente tende ao isolamento social, à depressão e à perda da vontade de prosseguir com o tratamento, tornando-se muito difícil para ele superar esses obstáculos.

\section{Fatores Relacionados aos Profissionais e ao Serviço}

Como foi possível identificar em 23 dos 57 artigos $22,24,25-28,30,32,34,36,40,44,46,47,52,55,56,67,72-74,78,79$, a falta de preparo de alguns profissionais, bem como a falta de estrutura de alguns serviços onde o tratamento era oferecido, impactou negativamente na adesão ao tratamento da tuberculose, visto que os pacientes não se sentiam acolhidos para levá-lo até o fim. A falta de educação e a grosseria de alguns profissionais também foi aspecto citado em alguns artigos.

Destaca-se, nessa categoria, a má relação entre profissional da saúde e paciente como fator importante de não adesão. Uma relação negativa implica falta de confiança do paciente no profissional que o está tratando. Essa desconfiança se soma ao fato de o paciente não se sentir acolhido pelo profissional e de pensar que não está recebendo atenção adequada nem apoio necessário. Todos esses fatores acabam influenciando na não adesão, juntamente com a falta de preparo por parte de alguns profissionais e com a falta de estrutura de determinados centros de saúde.

Watermeyer e Penn (2019) ${ }^{73}$ conduziram um estudo qualitativo, feito por entrevistas na África do Sul, que relacionou a não adesão ao tratamento à dificuldade de acesso aos serviços de saúde, seja pela falta de infraestrutura para o atendimento satisfatório dos pacientes, seja pela distância que impossibilitou o acesso, o que está em concordância com os achados dos outros estudos analisados neste trabalho $22,27,31,32,34,40,45,47,49,56,61,73,74,79$. Além disso, os problemas no sistema causaram uma descrença nos pacientes dessa localidade, o que extrapola os fatores vistos anteriormente. Este artigo também reforça a necessidade de um bom relacionamento entre profissionais de saúde e paciente, com confiança e respeito, o que evitaria o abandono.

Um estudo realizado com 27 pacientes com TB em unidades de Estratégia Saúde da Família (ESF), no município do Rio de Janeiro, concluiu que não adequar as rotinas e os horários às necessidades dos pacientes e às dificuldades que estes enfrentam causa um afastamento desses profissionais. Por outro lado, o interesse e dedicação dos profissionais para restabelecer a saúde dos pacientes, demonstrando preocupação, ajudam a criar laços, importantes para o seguimento do tratamento ${ }^{82}$.

\section{Fatores Relacionados ao Conhecimento}

Nessa categoria, enquadram-se os aspectos relacionados com a falta de conhecimento sobre a doença (tuberculose) e sobre o tratamento 
DOT, tanto dos profissionais de saúde quanto dos pacientes infectados.

Em todos os artigos em que foi abordada a questão do conhecimento, enfatizou-se que não ter informações acerca da doença e de seu tratamento foi um fator importante de não adesão ${ }^{19,23,28-30,32,34,36,37,40,48,50,54,61,65-67,72,74,79}$. O desconhecimento por parte dos profissionais resultou em descrença dos pacientes em relação ao tratamento ofertado. Estes perdiam a confiança na melhoria/cura. Ademais, a falta de conhecimento por parte dos pacientes ocasionou o abandono, pois estes não sabem sobre a importância de se terminar o tratamento adequadamente e não foram instruídos para isso. Desse modo, muitos deles, ao sentirem melhorias, não viam necessidade em prosseguir com a DOT.

Em um estudo conduzido na Índia, concluiu-se que não saber o causador da TB, como ela se dissemina, os sintomas dessa doença e se tem cura foram associados à não adesão ao tratamento. Além disso, não saber a duração do tratamento, os nomes e as cores dos medicamentos também são fatores relacionados ao abandono ${ }^{69}$.

Como limitações deste estudo, importa ressaltar que alguns dos artigos encontrados apresentaram uma população de estudo pequena. Além disso, foram incluídos estudos mais específicos, nos quais não se abordaram os fatores de não adesão de forma global, mas, sim, aspectos mais característicos da população e da região estudada. Assim, devem-se considerar as características loco-regionais de cada país e continente em que foram realizadas as pesquisas. Para tentar compensar essa limitação, utilizaram-se estudos de diferentes locais para a comparação dos resultados.

Tendo em vista os agravos que o não tratamento pode causar, é importante que sejam desenvolvidas políticas voltadas aos grupos de pacientes mais suscetíveis ao abandono. Considerando-se a logística, seria interessante disponibilizar a estratégia DOTS para outros centros de saúde ou criar novos centros, a fim de descentralizar o tratamento e facilitar o deslocamento dos pacientes. Também seria interessante criar políticas de intervenção que visem melhorias das condições sociais, através da promoção de moradias e do incremento dos cuidados em saúde. Será também relevante apostar na formação dos profissionais de saúde, com o intuito de aumentar seus conhecimentos bem como suas competências na interação terapêutica.

\section{CONCLUSÃO}

Este estudo possibilitou analisar as evidências disponíveis na literatura sobre os fatores relacionados à não adesão ao tratamento diretamente observado por pessoas com tuberculose pulmonar. Conclui-se que a multifacetada gama de aspectos associados a tal problema de pesquisa pode ser sintetizada em fatores relacionados ao paciente, a aspectos socioeconômicos, a fatores referentes ao tratamento, a fatores relacionados aos profissionais/serviço de saúde e a aspectos de conhecimento.

A não adesão ao tratamento da tuberculose merece atenção devido aos problemas que pode acarretar tanto para o paciente, quanto para o sistema de saúde, como para a sociedade. Tais comportamentos podem potencializar a proliferação de bactérias multirresistentes e elevar o número de acometidos, impactando a qualidade de vida e a gestão dos recursos em saúde. Recomenda-se que estratégias de enfrentamento sejam regularmente revistas e replanejadas, principalmente as destinadas aos grupos mais propensos à não adesão ao tratamento.

\section{REFERÊNCIAS}

1. Lima LM de, Schwartz E, Cardozo Gonzáles RI, Harter J, Lima JF de. O programa de controle da tuberculose em Pelotas/RS, Brasil: investigação de contatos intradomiciliares. Rev GaúchaEnferm. 2013;34(2):102-10.

2. Kozakevich GV, Da Silva, RM. Tuberculose: revisão de literatura. ArqCatarinenses Med. 2015; 4(44), 34-47.

3. World Health Organization 2018. Global tuberculosis report 2018. Genebra-Suíca; 2018. 2018; Disponível em: https://www.who.int/tb/publications/global_report/en/

4. BRASIL M da SFA. Tratamento Diretamente Observado da Tuberculose na Atenção Basica. 2011;2-172.

5. Rabahi MF, Da Silva Júnior JLR, Ferreira ACG, Tannus-Silva DGS, Conde MB. Tuberculosistreatment. J BrasPneumol. 2017;43(6):472-86.

6. Zegeye A, Dessie G, Wagnew F, Gebrie A, Islam SMS, Tesfaye $B$, et al. Prevalence and determinants of an- 
ti-tuberculosis treatment non-adherence in Ethiopia: A systematic review and meta-analysis. PLoS One. 2019;14(1).

7. Ferreira SMB, Silva AMC da, Botelho C. Abandono do tratamento da tuberculose pulmonar em Cuiabá - MT Brasil. J BrasPneumol. 2005;31(5):427-35.

8. Bhattacharya T, Ray S, Biswas P, Das D. Barriers to treatment adherence of tuberculosis patients: A qualitative study in West Bengal, India. Int J MedSciPublicHeal. $2018 ; 7(5): 1$.

9. Brasil. Brasil Livre da tuberculose. 2017;54. Disponível em: www.saude.gov.br/bvs

10. Kastien-Hilka T, Rosenkranz B, Bennett B, Sinanovic E, Schwenkglenks M. How to evaluate health-related quality of life and its association with medication adherence in pulmonary tuberculosis - designing a prospective observational study in South Africa. Front Pharmacol. 2016;7(MAIO).

11. Xun L, Ke $H$, Qin L, YongFeng J, ShengLan T. Are tuberculosis patients adherent to prescribed treatments in China? Resultsof a prospectivecohortstudy. InfectDisPoverty. $2016 ; 5(38)$.

12. Ercole FF, Melo LS de, Alcoforado CLGC. Integrative review versus systematic review. Reme Rev Min Enferm. $2014 ; 18(1)$.

13. Mendes KDS, Silveira RC de CP, Galvão CM. Revisão integrativa: método de pesquisa para a incorporação de evidências na saúde e na enfermagem. Texto Context - Enferm. 2008;17(4):758-64.

14. Santos CMDC, Pimenta CADM, Nobre MRC. A estratégia PICO para a construção da pergunta de pesquisa e busca de evidências. RevLatAm Enfermagem. 2007;15(3):508-11.

15. Ouzzani M, Hammady H, Fedorowicz Z, Elmagarmid A. Rayyan-a web and mobile app for systematic reviews. Syst Rev. 2016;5(1).

16. Ursi ES. Prevenção de lesões de pele no perioperatório: revisão integrativa da literatura. Rev Lat Am Enfermagem. $2005 ; 14(1): 2-127$.

17. Stillwell SB, Fineout-Overholt E, Melnyk BM, Williamson KM. Evidence-based practice, step by step: Asking the clinical question: A key step in Evidence-Based Practice. Am J Nurs. 2010;110(3):58-61.

18. Minayo MCS. O Desafio do conhecimento: pesquisa qualitativa em saúde. 12. ed. São Paulo: Hucitec, 2010. 407 p.

19. Caylà JA, Rodrigo T, Ruiz-Manzano J, Caminero JA, Vidal R, García JM, et al. Tuberculosis treatment adherence and fatality in Spain. Respir Res [Internet]. 2009;10:121. Disponível em: http://dx.doi.org/10.1186/1465-992110-121

20. Naidoo P, Dick J, Cooper D. Exploring tuberculosis patients' adherence to treatment regimens and prevention programs at a public health site. Qual Health Res [Internet]. $2009 ; 19(1): 55-70$. Available from: http://dx.doi. org/10.1177/1049732308327893
21. Xu W, Lu W, Zhou Y, Zhu L, Shen H, Wang J. Adherence to anti-tuberculosis treatment among pulmonary tuberculosis patients: A qualitative and quantitative study. BMC Health Serv Res. 2009;9:169.

22. Bagchi S, Ambe G, Sathiakumar N. Determinants of poor adherence to anti-tuberculosis treatment in Mumbai, India. Int J Prev Med. 2010;1(4):223-32.

23. Hasker E, Khodjikhanov M, Sayfiddinova S, Rasulova G, Yuldashova U, Uzakova G, et al. Why do tuberculosis patients default in Tashkent City, Uzbekistan? A qualitative study. Int J Tuberc Lung Dis. 2010;14(9):1132-9.

24. Kliiman K, Altraja A. Predictors and mortality associated with treatment default in pulmonary tuberculosis. Int J Tuberc Lung Dis. 2010;14(4):454-63.

25. Vijay S, Kumar P, Chauhan LS, Vollepore BH, Kizhakkethil UP, Rao SG. Risk factors associated with default among new smear positive TB patients treated under DOTS in India. PLoS One. 2010;5(4):e10043-e10043.

26. Burton NT, Forson A, Lurie MN, Kudzawu S, Kwarteng E, Kwara A. Factors associated with mortality and default among patients with tuberculosis attending a teaching hospital clinic in Accra, Ghana. Trans R SocTropMedHyg [Internet]. 2011;105(12):675-82. Disponível em: http://dx.doi.org/10.1016/j.trstmh.2011.07.017

27. Campani STA, Moreira J da S, Tietbohel CN. Fatores preditores para o abandono do tratamento da tuberculose pulmonar preconizado pelo Ministério da Saúde do Brasil na cidade de Porto Alegre (RS). J BrasPneumol [Internet]. 2011;37(6):776-82. Disponível em: http://www.scielo.br/scielo.php?script=sci_arttext\&pi$\mathrm{d}=\mathrm{S} 1806-37132011000600011$

28. Gupta S, Gupta S, Behera D. Reasons for interruption of anti-tubercular treatment as reported by patients with tuberculosis admitted in a tertiary care institute. Indian J Tuberc. $2011 ; 58(1): 11-7$.

29. Culqui DR, Munayco E. C V., Grijalva CG, Cayla JA, Horna-Campos O, Alva Ch. K, et al. Factors Associated With the Non-completion of Conventional Anti-Tuberculosis Treatment in Peru. Arch Bronconeumol (English Ed. 2012;48(5):150-5.

30. Finlay A, Lancaster J, Holtz TH, Weyer K, Miranda A, Van Der Walt M. Patient- and provider-level risk factors associated with default from tuberculosis treatment, South Africa, 2002: A case-control study. BMC Public Health [Internet]. 2012;12(1):56. Disponível em: http://dx. doi.org/10.1186/1471-2458-12-56

31. Ifebunandu NA, Ukwaja KN. Tuberculosis treatment default in a large tertiary care hospital in urban Nigeria: Prevalence, trend, timing and predictors. J Infect Public Health [Internet]. 2012;5(5):340-5. Disponível em: http://dx.doi.org/10.1016/j.jiph.2012.06.002

32. Kizub D, Ghali I, Sabouni R, Bourkadi JE, Bennani K, El Aouad R, et al. Qualitative study of perceived causes of tuberculosis treatment default among health care workers in Morocco. Int J TubercLungDis [Internet]. 2012;16(9):1214-20. Disponível em: http://dx.doi. org/10.5588/ijtld.11.0626 
33. Abreu GRF, Figueiredo MAA. Abandono do Tratamento da Tuberculose em Salvador (BA)-2005-2009. Rev Baiana Saúde Pública [Internet]. 2013;14(3):407-22. Disponível em: http://inseer.ibict.br/rbsp/index.php/rbsp/article/view/400/pdf_415

34. Belo EN, Orellana JDY, Levino A, Basta PC. Tuberculose nos municípios amazonenses da fronteira Brasil-Colômbia-Peru-Venezuela: situação epidemiológica e fatores associados ao abandono. Rev PanamSaludPublica/Pan Am J Public Heal [Internet]. 2013;34(5):321-9. Disponível em: <http:// www.scielosp.org/scielo.php?script $=$ sci_arttext\&pi$\mathrm{d}=$ S1020-49892013001100004>.

35. Bristow CC, Podewils LJ, Bronner LE, Bantubani N, Van Der Walt M, Peters A, et al. TB tracer teams in South Africa: Knowledge, practices and challenges of tracing TB patients to improve adherence. BMC Public Health [Internet]. 2013;13(1):801. Disponívelem: http://dx. doi.org/10.1186/1471-2458-13-801

36. Mehra D, Kaushik RM, Kaushik R, Rawat J, Kakkar R. Initial default among sputum-positive pulmonary TB patients at a referral hospital in Uttarakhand,India. Trans $R$ SocTropMedHyg [Internet]. 2013;107(9):558-65. Disponível em: http://dx.doi.org/10.1093/trstmh/trt065

37. Slama K, Tachfouti N, Obtel M, Nejjari C. Factors associated with treatment default by tuberculosis patients in Fez, Morocco. EastMediterrHeal J. 2013;19(08):687-93.

38. Cherkaoui I, Sabouni R, Ghali I, Kizub D, Billioux AC, Bennani $K$, et al. Treatment default amongst patients with tuberculosis in urban Morocco: Predicting and explaining default and post-default sputum smear and drug susceptibility results. PLoSOne [Internet]. 2014;9(4):e93574-e93574. Disponível em: http://dx. doi.org/10.1371/journal. pone.0093574

39. Costa KB, Silva CEF, Martins AF. Características Clínicas e epidemiológicas de pacientes com tuberculose na cidade com a maior incidência da doença no Brasil. ClinBiomed Res [Internet]. 2014;34(1):40-6. Disponível em: http://www.seer.ufrgs.br/index.php/hcpa/article/download/43291/28770

40. Ibrahim LM, Hadejia IS, Nguku $P$, Dankoli $R$, Waziri NE, Akhimien MO, et al. Factors associated with interruption of treatment among pulmonary tuberculosis patients in plateau state, Nigeria. 2011. Pan AfrMed J [Internet]. 2014;17:78. Disponível em: http://dx.doi. org/10.11604/pamj.2014.17.78.3464

41. Rondags A, Himawan AB ud., Metsemakers JF m., Kristina TN u. Factors influencing non-adherence to tuberculosis treatment in Jepara, central Java, Indonesia. Southeast Asian J Trop Med Public Health. 2014;45(4):859-68.

42. Silva PDF, Moura GS, Caldas A de JM. Fatores associados ao abandono do tratamento da tuberculose pulmonar no Maranhão, Brasil, no período de 2001 a 2010. CadSaude Publica. 2014;30(8):1745-54.

43. Zhang Q, Gaafer M, El Bayoumy I. Determinants of default from pulmonary tuberculosis treatment in Kuwait. Sci World J. 2014;2014:672825.
44. Chida N, Ansari Z, Hussain H, Jaswal M, Symes S, Khan AJ, et al. Determinants of default from tuberculosis treatment among patients with drug-susceptible tuberculosis in Karachi, Pakistan: A mixed methods: Study. PLoSOne [Internet]. 2015;10(11):e0142384-e0142384. Disponível em: http://dx.doi.org/10.1371/journal. pone. 0142384

45. De Andrade EDT, Hennington ÉA, De Siqueira HR, Rolla VC, Mannarino C. Perspectives of patients, doctors and medical students at a public university hospital in rio de janeiro regarding tuberculosis and therapeutic adherence. PLoSOne. 2015;10(9).

46. Sitienei J, Kipruto $H$, Mansour O, Ndisha M, Hanson C, Wambu $R$, et al. Correlates of default from anti-tuberculosis treatment: A case study using Kenya's electronic data system. Int J Tuberc Lung Dis. 2015;19(9):1051-6.

47. Tesfahuneygn G, Medhin G, Legesse M. Adherence to Anti-tuberculosis treatment and treatment outcomes among tuberculosis patients in Alamata District, northeast Ethiopia. BMC Res Notes. 2015;8(1).

48. Theron G, Peter J, Zijenah L, Chanda D, Mangu C, Clowes $P$, et al. Psychological distress and its relationship with non-adherence to TB treatment: A multicentre study. BMC InfectDis [Internet]. 2015;15(1):253. Disponível em: http://dx.doi.org/10.1186/s12879-015-0964-2

49. Ali AOA, Prins MH. Patient non adherence to tuberculosis treatment in Sudan: Socio demographic factors influencing non adherence to tuberculosis therapy in Khartoum State. Pan AfrMed J [Internet]. 2016;25.

50. Anduaga Beramendi A, Maticorena Quevedo J, Beas R, Chanamé Baca $D$, Veramendi M, Wiegering Rospigliosi $A$, et al. Factores de riesgo para el abandono del tratamiento de tuberculosis pulmonar sensible en un establecimiento de salud de atención primaria, Lima, Perú. Acta Médica Peru [Internet]. 2016;33(1):21-8. Disponível em: <http://repebis.upch.edu.pe/articulos/acta.med. per/v33n1/a5.pdf>.

51. Choi $H$, Chung $H$, Muntaner $C$, Lee $M$, Kim $Y$, Barry CE, et al. The impact of social conditions on patient adherence to pulmonary tuberculosis treatment. Int J Tuberc Lung Dis. $2016 ; 20(7): 948-54$.

52. Fagundez G, Perez-Freixo H, Eyene J, Momo JC, Biyé $\mathrm{L}$, Esono $\mathrm{T}$, et al. Treatment adherence of tuberculosis patients attending two reference units in Equatorial Guinea. PLoSOne [Internet]. 2016;11(9):e0161995e0161995. Disponível em: http://dx.doi.org/10.1371/ journal.pone.0161995

53. Heemanshu A, Satwanti K. Determinants of lost to follow up during treatment among tuberculosis patients in deIhi. Int J Med Res Heal Sci. 2016;5(1):145-52.

54. Choowong J, Tillgren $P$, Söderbäck M. Thai people living with tuberculosis and how they adhere to treatment: A grounded theory study. Nurs Heal Sci [Internet]. $2017 ; 19(4): 436-43$.

55. Da Silva RD, De Luna FDT, De Araújo AJ, Camêlo ELS, Bertolozzi MR, Hino $P$, et al. Patients' perception regarding the influence of individual and social vulnerabilities on the 
adherence to tuberculosis treatment: A qualitative study. BMC Public Health [Internet]. 2017;17(1):725. Disponível em: http://dx.doi.org/10.1186/s12889-017-4752-3

56. GugssaBoru C, Shimels T, Bilal AI. Factors contributing to non-adherence with treatment among TB patients in Sodo Woreda, Gurage Zone, Southern Ethiopia: A qualitative study. J Infect Public Health [Internet]. 2017;10(5):527-33. Disponível em: http://dx.doi.org/10.1016/j.jiph.2016.11.018

57. Harling G, Lima Neto AS, Sousa GS, Machado MMT, Castro MC. Determinants of tuberculosis transmission and treatment abandonment in Fortaleza, Brazil. BMC Public Health [Internet]. 2017;17(1). Disponívelem: file:/// pubmed/28545423

58. Nunes C, Duarte R, Veiga AM, Taylor B. Who are the patients that default tuberculosis treatment? - Space matters! Epidemiol Infect [Internet]. 2017;145(6):11304. Available from: http://dx.doi.org/10.1017/ S0950268816003307

59. Soares MLM, Amaral NAC do, Zacarias ACP, Ribeiro LK de NP. Aspectos sociodemográficos e clínico-epidemiológicos do abandono do tratamento de tuberculose em Pernambuco, Brasil, 2001-2014. Epidemiol e Servsaude Rev do Sist Único Saude do Bras [Internet]. 2017;26(2):36978. Disponível em: http://www.scielo.br/scielo.php?script $=$ sci_arttext\&pid $=$ S2237-96222017000200369

60. Tola HH, Garmaroudi G, Shojaeizadeh D, Tol A, Yekaninejad MS, Ejeta LT, et al. The Effect of Psychosocial Factors and Patients' Perception of Tuberculosis Treatment Non-Adherence in Addis Ababa, Ethiopia. Ethiop J Health Sci. 2017;27(5):447-58.

61. Wanyonyi AW, Wanjala PM, Githuku J, Oyugi E, Kutima $\mathrm{H}$. Factors associated with interruption of tuberculosis treatment among patients in Nandi County, Kenya 2015. Pan AfrMed J [Internet]. 2017;28:11. Disponível em: http://dx.doi.org/10.11604/pamj.supp.2017.28.1.9347

62. De Oliveira SM, Altmayer S, Zanon M, Sidney-Filho LA, Moreira ALS, de Tarso Dalcin P, et al. Predictors of noncompliance to pulmonary tuberculosis treatment: An insight from South America. PLoSOne. 2018;13(9).

63. Diallo A, Dahourou DL, Dah TTE, Tassembedo S, Sawadogo R, Meda N. Factors associated with tuberculosis treatment failure in the central east health region of Burkina Faso. Pan AfrMed J [Internet]. 2018;30:293. Disponível em: http://dx.doi.org/10.11604/ pamj.2018.30.293.15074

64. García P, Sanchez J, Mora J, Ronda E. Assessment of 16-year retrospective cohort study of factors associated with non-compliance with a tuberculosis contact tracingprogramme at a Spanish hospital. J EvalClinPract. 2018;24(4):758-66.

65. Ruru Y, Matasik M, Oktavian A, Senyorita R, Mirino Y, Tarigan $\mathrm{LH}$, et al. Factors associated with non-adherence during tuberculosis treatment among patients treated with DOTS strategy in Jayapura, Papua Province, Indonesia. Glob Health Action [Internet]. 2018;11(1). Disponível em: file:///pubmed/30394200
66. Chakrabartty A, Basu P, Ali KM, Ghosh D. Tuberculosis related stigma attached to the adherence of Directly Observed Treatment Short Course (DOTS) in West Bengal, India. Indian J Tuberc. 2019;66(2):259-65.

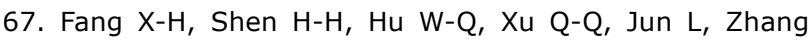
$Z-P$, et al. Prevalence of and Factors Influencing Anti-Tuberculosis Treatment Non-Adherence Among Patients with Pulmonary Tuberculosis: A Cross-Sectional Study in Anhui Province, Eastern China. Med Sci Monit [Internet]. 2019;25:1928-35. Disponível em: http://dx.doi. org/10.12659/MSM.913510

68. Jose J, Helen George J, Vignesh R, Chetty S, Ganesan R. Medication adherence to anti tuberculosis treatment among tuberculosis patients in an urban private tertiary referral hospital: a prospective cross sectional study. Res Artic Jaisy al World J Pharm Res. 2019;8(6):599.

69. Aguilar JP, Arriaga MB, Rodas MN, Martins Netto E. Smoking and pulmonary tuberculosis treatment failure: a case-control study. J BrasPneumol. 2019;45(2):e20180359. Disponível em: <http:// www.scielo.br/scielo.php?script $=$ sci_arttext\&pi$\mathrm{d}=\mathrm{S} 1806-37132019000200205 \& \mathrm{lng}=\mathrm{en \&} \mathrm{nrm}=\mathrm{iso}$ > .

70. Holden IK, Lillebaek T, Seersholm N, Andersen PH, Wejse C, Johansen IS. Predictors for Pulmonary Tuberculosis Treatment Outcome in Denmark 2009-2014. Sci Rep. $2019 ; 9(1)$.

71. Watermeyer J, Penn C. Community perspectives on tuberculosis care in rural South Africa. Heal Soc Care Community. 2019;27(1):182-90.

72. Dueñes M, Cardona D. Factors related to treatment adherence in patients with tuberculosis in Pereira, Colombia, 2012-2013. Biomedica. 2016;36(3):423-31.

73. Negandhi H, Tiwari R, Sharma A, Nair R, Zodpey S, Allam RR, et al. Rapid assessment of facilitators and barriers related to the acceptance, challenges and community perception of daily regimen for treating tuberculosis in India. Glob Health Action [Internet]. 2017;10(1):1290315. Disponívelem: http://dx.doi.org/ 10.1080/16549716.2017.1290315.

74. Woimo TT, Yimer WK, Bati T, Gesesew HA. The prevalence and factors associated for anti-tuberculosis treatment non-adherence among pulmonary tuberculosis patients in public health care facilities in South Ethiopia: a cross-sectional study. BMC Public Health. 2017;17(1):269.

75. De Seixas Maciel EMG, De Souza Amancio J, De Castro DB, Braga JU. Social determinants of pulmonary tuberculosis treatment non-adherence in Rio de Janeiro, Brazil. PLoS One [Internet]. 2018;13(1):e0190578e0190578. Disponível em: http://dx.doi.org/10.1371/ journal.pone.0190578

76. Orlandi GM, Pereira ÉG, Biagolini REM, França FO de $S$, Bertolozzi MR. Social incentives for adherence to tuberculosis treatment. Rev Bras Enferm. 2019 Sep; 72(5):1182-8.

77. Reis-Santos B, Shete $P$, Bertolde A, Sales CM, Sanchez MN, Arakaki-Sanchez D, et al. Tuberculosis in Brazil and 
cash transfer programs: A longitudinal database study of the effect of cash transfer on cure rates. PLoS One. $2019 ; 14(2)$.

78. Mesfin MM, Newell JN, Walley JD, Gessessew A, Tesfaye $T$, Lemma $F$, et al. Quality of tuberculosis care and its association with patient adherence to treatment in eight Ethiopian districts. Health PolicyPlan. $2009 ; 24(6): 457-66$.

79. Rao NA, Anwer T, Saleem M. Magnitude of initial default in pulmonary tuberculosis. J Pak Med Assoc. $2009 ; 59(4): 223-4$.
80. Marx FM, Dunbar R, Hesseling AC, Enarson DA, Fielding $K$, Beyers N. Increased risk of default among previously treated tuberculosis cases in the Western Cape Province, South Africa. Int J TubercLungDis [Internet]. 2012;16(8):105965. Disponível em: http://dx.doi.org/10.5588/ijtld.11.0506

81. Marquieviz J, Alves I dos S, Neves EB, Ulbricht L. A estratégia de Saúde da Família no controle da tuberculose em Curitiba (PR). Cienc e Saude Coletiva. 2013;18(1):265-71.

82. Linhares SR dos S, Paz EPA. A vivência do tratamento de tuberculose em unidades de Saúde da Família. Esc Anna Nery. $2020 ; 24(2)$.

\section{Contribuição individual dos autores:}

Autores 1, 2, 3 e 6: concepção e planejamento do estudo; obtenção, análise e interpretação dos dados; redação e aprovação final da versão publicada.

Autores 4 e 5: obtenção, análise e interpretação dos dados; redação e aprovação final da versão publicada.

\section{Agradecimentos:}

À Universidade Federal de Alfenas, UNIFAL-MG.

Declaração de conflito de interesses: Nada a declarar. Não há conflitos de interesse políticos, financeiros associados a patentes ou propriedade, provisão de materiais, insumos ou equipamentos utilizados no estudo.

\section{Fontes de financiamento:}

A pesquisa não recebeu financiamento para a sua realização.

Corresponding author:

Greyce Khoury Mansour.

greycemansour@gmail.com

Editor:

Prof. Dr Felipe Villela Gomes

Recebido: $17 / 07 / 2020$

Aprovado: $26 / 10 / 2020$ 